\title{
A Note on the Efficiency Based Reliability Measures for Heterogeneous Populations
}

\author{
Ji Hwan Cha ${ }^{1, a}$ \\ ${ }^{a}$ Department of Statistics, Ewha Womans University
}

\begin{abstract}
In many cases, populations in the real world are composed of different subpopulations. Furthermore, in addition to the heterogeneity in the lifetimes of items, there also could be the heterogeneity in the efficiency or performance of items. In this case, the reliability measures should be defined in a different way. In this article, we consider the mixture of stochastically ordered subpopulations. Efficiency based reliability measures are defined when the performance of items in the subpopulations has different levels. Discrete and continuous mixing models are studied. The concept of the association between the lifetime and the performance of items in subpopulations is defined. It is shown that the consideration of efficiency can change the shape of the mixture failure rate dramatically especially when the lifetime and the performance of items in subpopulations are negatively associated. Furthermore, the modelling method proposed in this paper is applied to the case when the stress levels of the operating environment of items are different.
\end{abstract}

Keywords: Heterogeneous population, failure rate, efficiency, transformed time scale, association.

\section{Introduction}

Most populations encountered in the real world are heterogeneous. In reliability context, items with different quality may be produced due to, for example, defective resources and components, human errors, unstable production environment, etc. In this case, two subpopulations of strong and weak components usually constitute the mixture population (Jensen and Petersen, 1982). It is also well known that the continuous mixture is an effective tool for modeling population heterogeneity due to a randomly changing production environment and other causes of 'random effects' (Finkelstein, 2008). Therefore, these models are important in various reliability engineering applications.

The mixture failure rate for two ordered subpopulations was intensively studied in the literature. For instance, Gupta and Warren (2001) show that the mixture of two gamma distributions with increasing failure rates (with the same scale parameter) can result either in the increasing mixture failure rate or in the modified bathtub(MBT) mixture failure rate (it first increases and then behaves like a bathtub failure rate). Similar shapes occur for the mixtures of two Weibull distributions with increasing failure rates (Jiang and Murthy, 1995). Navarro and Hernandez (2004) state that the mixture failure rate of two truncated normal distributions, depending on parameters involved, can also be increasing, BT(bathtub)-shaped or MBT-shaped. Block et al. (2003) give explicit conditions which describe the possible shapes of the mixture failure rate for two increasing linear failure rates.

In many cases, the performance of items in the population may have different levels. In this case, the reliability measures should be defined in a different way and the ageing property should be obtained considering the efficiency of the items. In this article, we consider the mixture of stochastically

This work was supported by Priority Research Centers Program through the National Research Foundation of Korea(NRF) funded by the Ministry of Education, Science and Technology(2009-0093827).

${ }^{1}$ Associate Professor, Department of Statistics, Ewha Womans University, Seoul 120-750, Korea. E-mail: jhcha@ewha.ac.kr 
ordered subpopulations, which is defined in terms of the ordering of failure rates of subpopulations. Efficiency based reliability measures are defined for discrete and continuous mixing models. Furthermore, the modelling method proposed in this paper is applied to the case when the operating environments of items are different.

This paper is constructed as follows. In Section 2, we consider the case of discrete mixing model. Performance based reliability measure for a subpopulation is defined and, based on it, the mixture population failure rate function is obtained. The concept of the association between the lifetime and the performance of items in subpopulations is defined and it is shown that the consideration of the efficiency can change the shape of the mixture failure rate dramatically especially when the lifetime and the performance of items in subpopulations are negatively associated. In Section 3, the foregoing discussions are extended to the case of continuous mixing. In Section 4, the modelling method discussed in the previous section is applied to the case when the stress levels of the operating environment are different. In Section 5, some possible practical applications of the proposed methods are proposed.

\section{Discrete Mixing}

We will consider the mixture of stochastically ordered subpopulations. Thus, for convenience, we will call the two subpopulations 'strong' and 'weak' subpopulations. In this section, we consider the case of $n=2$ subpopulations (that can be generalized to the arbitrary finite $n$ ). Denote the lifetime of a component from the strong subpopulation by $T_{1}$ and its absolutely continuous Cdf, pdf and the failure rate function by $F_{1}(t), f_{1}(t)$ and $\lambda_{1}(t)$, respectively. Similarly, the lifetime, the Cdf, pdf and the failure rate function of a weak component are $T_{2}, F_{2}, f_{2}(t)$ and $\lambda_{2}(t)$, accordingly. Formal definitions of strong and weak subpopulations will be given after introducing the necessary notations. The initial $(t=0)$ composition of our mixed population is as follows: the proportion of strong items is $\pi$, whereas the proportion of weak items is $1-\pi$, which means that the distribution of the discrete frailty $Z$ with realizations 1 and 2 in this case is

$$
\pi(z)= \begin{cases}\pi, & z=1 \\ 1-\pi, & z=2\end{cases}
$$

and 1,2, correspond to the strong and the weak subpopulations, respectively. The mixture (population) survival function is

$$
\bar{F}_{m}(t)=\pi \bar{F}_{1}(t)+(1-\pi) \bar{F}_{2}(t) .
$$

Then the mixture (observed) failure rate is

$$
\lambda_{m}(t)=\frac{\pi f_{1}(t)+(1-\pi) f_{2}(t)}{\pi \bar{F}_{1}(t)+(1-\pi) \bar{F}_{2}(t)}=\pi(t) \lambda_{1}(t)+(1-\pi(t)) \lambda_{2}(t),
$$

where the time-dependent probabilities are

$$
\pi(t)=\frac{\pi \bar{F}_{1}(t)}{\pi \bar{F}_{1}(t)+(1-\pi) \bar{F}_{2}(t)}, \quad 1-\pi(t)=\frac{(1-\pi) \bar{F}_{2}(t)}{\pi \bar{F}_{1}(t)+(1-\pi) \bar{F}_{2}(t)} .
$$

Assume now that the populations are ordered (and therefore, the weak and the strong subpopulations are defined accordingly) in the sense of the failure rate ordering:

$$
\lambda_{1}(t) \leq \lambda_{2}(t), \quad t \geq 0 .
$$


The above reliability measures are valid only when the 'efficiency' of items between different subpopulations is the same. However, we consider the situation when "the larger (or smaller) values of $Z$ correspond to the lower efficiency of items". For example, when compared with a strong item, a weak item's efficiency is 0.5 times of that of a strong component, which implies that it takes twice the time length for a weak item to perform the same amount of work as a strong item does. In this situation, for a strong item, it takes 1 unit of time to perform 1 unit amount of work, whereas, for a weak item, it takes 2 units of time to perform 1 unit amount of work. That is, the absolute lifetime, in this situation, has little meaning. Therefore, in this heterogeneous setting, different time scales depending on the corresponding subpopulations need to be adopted.

Now, the above reasoning is mathematically formulated. Let $e_{1}$ represent the efficiency of the subpopulation with frailty $Z=1$ (strong subpopulation) and let $e_{2}$ be that of subpopulation with frailty $Z=2$ (weak subpopulation). Without a loss of generality, in the following discussion, we may set $e_{1} \equiv 1$ unless otherwise specified. This implies that, if a strong item operates for $t$ unit of time, then it performs $t$ unit amount of work, whereas, a weak item performs $e_{2} \cdot t$ unit amount of work for the same time period. If, for example, the item under consideration is a production system, then $e_{2}=0.5$ implies that the number of products which a weak system produces corresponds to only $50 \%$ of that a strong system produces for the same time length.

Practically, $e_{2}$ can be estimated in the following manner. The amount of work performed by the weak item $y_{i}$ (unit amount of work) is observed at each fixed time point $t_{i}, i=1,2, \ldots, m, t_{1}<t_{2}<$ $\cdots<t_{m}$. Then based on the observation $\left(y_{i}, t_{i}\right), i=1,2, \ldots, m$, a simple linear regression model $E(y)=e_{2} \cdot t$ which passes through the origin can be fitted. Then the estimator for $e_{2}$ can be obtained without difficulty.

Now, the concept of the association between the subpopulation lifetime and the corresponding efficiency is defined in the following definition.

\section{Definition 1. (Association between the Lifetime and the Performance: Discrete Case)}

For the case of $n=2$, if $e_{1}>e_{2}\left(e_{1}<e_{2}\right)$, then the lifetime and the efficiency are positively (negatively) associated.

The above efficiency concepts are defined 'relatively' and thus there should be a baseline. As we set $e_{1} \equiv 1$, the efficiency of the strong subpopulation is the 'baseline'. Under this setting, the time needed for a strong item to perform $t$ unit amount of work is $t$ and, on the other hand, that for a weak item is $t / e_{2}$. Therefore, the probability that an item from subpopulation $i$ will perform $t$ unit amount of work without failure is defined by

$$
\bar{F}_{C}(t, i)=P\left(T_{i}>c_{i}(t)\right)=\bar{F}_{i}\left(c_{i}(t)\right)=\exp \left\{-\int_{0}^{c_{i}(t)} \lambda_{i}(u) d u\right\},
$$

where $c_{i}(t) \equiv\left(1 / e_{i}\right) t, i=1,2$. Now, $c_{i}(t)$ can be interpreted as the 'transformed time scale' for the subpopulation $i, i=1,2$, that is, the lifetime of $c_{i}(t)$ corresponds to the lifetime which yields $t$ unit amount of work, $i=1,2$. Thus the distribution in (2.1) can be understood as the lifetime distribution defined in the transformed time scale. As the distribution in (2.1) is a lifetime distribution, the corresponding failure rate function can be defined as

$$
\lambda_{C}(t, i) \equiv \frac{f_{C}(t, i)}{\bar{F}_{C}(t, i)}
$$


where $f_{C}(t, i)=-d \bar{F}_{C}(t, i) / d t, i=1,2$. Then the failure rate in (2.2) can be interpreted via:

$$
\begin{aligned}
\lambda_{C}(t, i) \cdot \Delta t \approx P & \text { (The item will fail during working additional } \Delta t \\
& \text { unit amount of work } \mid \text { The item has worked } t \text { unit amount of work). }
\end{aligned}
$$

Then the mixture survival function based on the transformed time scale is given by

$$
\bar{F}_{m C}(t)=\pi \bar{F}_{C}(t, 1)+(1-\pi) \bar{F}_{C}(t, 2),
$$

and the mixture (observed) failure rate is

$$
\lambda_{m C}(t)=\pi_{C}(t) \lambda_{C}(t, 1)+\left(1-\pi_{C}(t)\right) \lambda_{C}(t, 2)
$$

where

$$
\lambda_{C}(t, i)=\frac{f_{C}(t, i)}{\bar{F}_{C}(t, i)}=c_{i}^{\prime}(t) \lambda_{i}\left(c_{i}(t)\right), \quad i=1,2
$$

and the time-dependent probabilities are

$$
\pi_{C}(t)=\frac{\pi \bar{F}_{C}(t, 1)}{\pi \bar{F}_{C}(t, 1)+(1-\pi) \bar{F}_{C}(t, 2)}, \quad 1-\pi_{C}(t)=\frac{(1-\pi) \bar{F}_{C}(t, 2)}{\pi \bar{F}_{C}(t, 1)+(1-\pi) \bar{F}_{C}(t, 2)} .
$$

Observe that if the lifetime and efficiency are positively associated and the failure rate functions of the subpopulations are non-decreasing, then the stochastic order between the lifetimes of the subpopulations is preserved as $c_{1}(t)<c_{2}(t)$ and $c_{2}^{\prime}(t)>1$. On the other hand, if the lifetime and efficiency are negatively associated, then the stochastic order is not necessarily preserved.

Proposition 1. If the lifetime and efficiency are positively associated and the failure rate functions of the subpopulations are non-decreasing, then the stochastic order between the lifetimes of the subpopulations is preserved under the transformed time scale.

This result implies that when the lifetime and efficiency are negatively associated, the efficiency based approach can change the shapes of the mixture failure rate dramatically. See the following example for detailed illustrations.

Example 1. Suppose that $\lambda_{1}(t)=\lambda_{1}, t \geq 0$ and $\lambda_{2}(t)=\lambda_{2}, t \geq 0, \lambda_{2}>\lambda_{1}$. Then the ordinary mixture failure rate $\lambda_{m}(t)$ is obtained by

$$
\begin{aligned}
\lambda_{m}(t) & =\pi(t) \lambda_{1}(t)+(1-\pi(t)) \lambda_{2}(t) \\
& =\frac{\pi e^{-\lambda_{1} t}}{\pi e^{-\lambda_{1} t}+(1-\pi) e^{-\lambda_{2} t}} \lambda_{1}+\frac{(1-\pi) e^{-\lambda_{2} t}}{\pi e^{-\lambda_{1} t}+(1-\pi) e^{-\lambda_{2} t}} \lambda_{2},
\end{aligned}
$$

which is a decreasing function. Now $\lambda_{C}(t, 1) \equiv \lambda_{1}(t)$ and, as $c_{2}(t)=\left(1 / e_{2}\right) t$,

$$
\lambda_{C}(t, 2) \equiv c_{2}^{\prime}(t) \lambda_{2}\left(c_{2}(t)\right)=\left(\frac{1}{e_{2}}\right) \lambda_{2}, \quad t \geq 0
$$

Therefore, $\lambda_{m C}(t)$ in (2.3) is obtained by

$$
\lambda_{m C}(t)=\frac{\pi e^{-\lambda_{1} t}}{\pi e^{-\lambda_{1} t}+(1-\pi) e^{-\left(\frac{\lambda_{2}}{e_{2}}\right) t}} \lambda_{1}+\frac{(1-\pi) e^{-\left(\frac{\lambda_{2}}{e_{2}}\right) t}}{\pi e^{-\lambda_{1} t}+(1-\pi) e^{-\left(\frac{\lambda_{2}}{e_{2}}\right) t}}\left(\frac{\lambda_{2}}{e_{2}}\right) .
$$




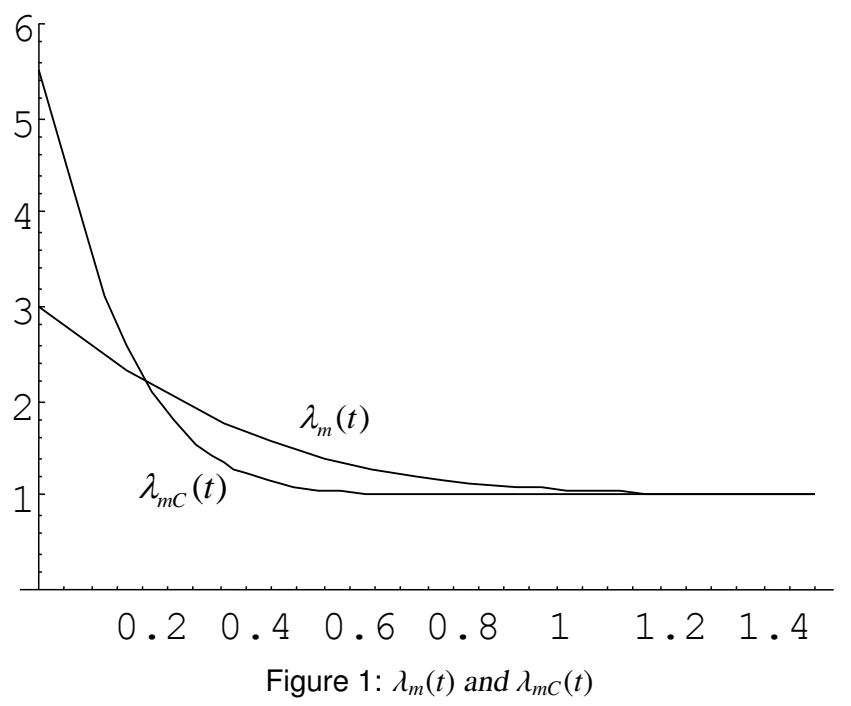

(Case I: Positive Association) Let $\lambda_{1}=1.0, \lambda_{2}=5.0$ and $e_{2}=0.5$. In this case, the graphs for $\lambda_{m}(t)$ and $\lambda_{m C}$ are given in Figure 1. In this case, both of mixture failure rates decrease even though each subpopulation has a constant failure rate. Furthermore, it can be seen that they approach the failure rates of stronger subpopulations, respectively. This phenomenon can be well explained by the property that the weakest items are dying out first.

(Case II: Negative Association) Let $\lambda_{1}=1.0, \lambda_{2}=5.0$ and $e_{2}=5.0$. In this case, $\lambda_{C}(t, 2)=$ $\left(1 / e_{2}\right) \lambda_{2}=1.0$ and therefore $\lambda_{m C}(t)=1.0, t \geq 0$. Thus, in this case, the consideration of the efficiency dramatically changes the shapes of the mixture failure rates(DFR to CFR).

Remark 1. The setting with two subpopulations discussed in this section can be straightforwardly extended to the case of $n>2$ stochastically ordered subpopulations. Let $F_{i}(t), f_{i}(t)$ and $\lambda_{i}(t)$ be the Cdf, pdf and failure rate function of subpopulation $i$, respectively, $i=1,2, \ldots, n$. Furthermore, let $\pi_{i}$ be the proportion of the $i$ th subpopulation, $i=1,2, \ldots, n$, where $\sum_{i=1}^{n} \pi_{i}=1$. Now, as before, the subpopulations are assumed to be stochastically ordered as:

$$
\lambda_{1}(t) \leq \lambda_{2}(t) \leq \cdots \leq \lambda_{n}(t)
$$

Then it can be easily verified that the mixture (observed) failure rate is given by

$$
\lambda_{m}(t)=\sum_{i=1}^{n} \pi_{i}(t) \lambda_{i}(t)
$$

where the time-dependent probabilities are

$$
\pi_{i}(t)=\frac{\pi_{i} \bar{F}_{i}(t)}{\sum_{j=1}^{n} \pi_{j} \bar{F}_{j}(t)}, \quad i=1,2, \ldots, n .
$$

Defining the efficiency of subpopulation $i, e_{i}$, and $c_{i}(t) \equiv\left(1 / e_{i}\right) t, i=1,2, \ldots, n$, the corresponding survival function $\bar{F}_{C}(t, i)$ and the failure rate function $\lambda_{C}(t, i)$ under the transformed time scale can be 
similarly defined. Then, as before, the mixture (observed) failure rate on the transformed time scale is given by

$$
\lambda_{m C}(t)=\sum_{i=1}^{n} \pi_{C}(t, i) \lambda_{C}(t, i)
$$

where

$$
\lambda_{C}(t, i) \equiv c_{i}^{\prime}(t) \lambda_{i}\left(c_{i}(t)\right)
$$

and the time-dependent probabilities are

$$
\pi_{C}(t, i)=\frac{\pi_{i} \bar{F}_{C}(t, i)}{\sum_{j=1}^{n} \pi_{j} \bar{F}_{C}(t, j)}, \quad i=1,2, \ldots, n .
$$

\section{Continuous Mixing}

Now we consider the continuous mixing case. We assume the following heterogeneous structure:

$$
\bar{F}_{m}(t)=\int_{0}^{\infty} \bar{F}(t, z) \pi(z) d z, \quad F_{m}(t)=\int_{0}^{\infty} F(t, z) \pi(z) d z, \quad f_{m}(t)=\int_{0}^{\infty} f(t, z) \pi(z) d z,
$$

where $F(t, z)$ and $f(t, z)$ are the Cdf and the pdf of subpopulations indexed by the frailty parameter $Z$ and $\pi(z)$ is the pdf of $Z$ with support in $[0, \infty)$. The general support $[a, b), 0 \leq a<b \leq \infty$, can be considered as well. Then the mixture (the observed or the population) failure rate $\lambda_{m}(t)$ is defined as

$$
\lambda_{m}(t)=\frac{\int_{0}^{\infty} f(t, z) \pi(z) d z}{\int_{0}^{\infty} \bar{F}(t, z) \pi(z) d z}=\int_{0}^{\infty} \lambda(t, z) \pi(z \mid t) d z,
$$

where the conditional density (on condition that the item did not fail in $[0, t)$ ) is

$$
\pi(z \mid t) \equiv \pi(z) \frac{\bar{F}(t, z)}{\int_{0}^{\infty} \bar{F}(t, z) \pi(z) d z} .
$$

Let our subpopulations be ordered in the sense of the failure rate ordering

$$
\lambda\left(t, z_{1}\right) \leq \lambda\left(t, z_{2}\right), \quad z_{1}<z_{2}, \forall z_{1}, z_{2} \in[0, \infty), t \geq 0,
$$

and the efficiency function of the subpopulation with frailty $Z=z$ be $e_{z}$.

Now, as in the case of the discrete case, the association between the subpopulation lifetime and the corresponding efficiency can be defined as follows.

\section{Definition 2. (Association between the Lifetime and the Performance: Continuous Case)}

For the case of continuous mixing model, if $e_{z_{1}}>e_{z_{2}}$, for any $z_{1}$ and $z_{2}$ such that $z_{1}<z_{2}\left(e_{z_{1}}<e_{z_{2}}\right.$, for any $z_{1}$ and $z_{2}$ such that $z_{1}<z_{2}$ ), then the lifetime and the performance are positively (negatively) associated.

As in the Discrete Case, in the following discussions, we assume that the baseline subpopulation is the strongest one unless otherwise specified. Thus we let $e_{0}=1$. Now we define the 'transformed' time scales for the subpopulations:

$$
c_{z}(t) \equiv\left(\frac{1}{e_{z}}\right) t, \quad z \geq 0, t \geq 0 .
$$


Then the survival function for the subpopulation with $Z=z$ based on the transformed time scale is

$$
\bar{F}_{C}(t, z)=\exp \left\{-\int_{0}^{c_{z}(t)} \lambda(u, z) d u\right\}
$$

where $\lambda(u, z)=f(u, z) / \bar{F}(u, z)$, and the corresponding failure rate is given by

$$
\lambda_{C}(t, z)=\frac{f_{C}(t, z)}{\bar{F}_{C}(t, z)}=c_{z}^{\prime}(t) \lambda\left(c_{z}(t), z\right)
$$

where $f_{C}(t, z)=-\partial \bar{F}_{C}(t, z) / \partial t$. Then

$$
\bar{F}_{m C}=\int_{0}^{\infty} \bar{F}_{C}(t, z) \pi(z) d z, \quad F_{m C}(t)=\int_{0}^{\infty} F_{C}(t, z) \pi(z) d z, \quad f_{m C}(t)=\int_{0}^{\infty} f_{C}(t, z) \pi(z) d z
$$

and $\lambda_{m C}(t)$ is defined as

$$
\lambda_{m C}(t)=\frac{\int_{0}^{\infty} f_{C}(t, z) \pi(z) d z}{\int_{0}^{\infty} \bar{F}_{C}(t, z) \pi(z) d z}=\int_{0}^{\infty} \lambda_{C}(t, z) \pi_{C}(z \mid t) d z
$$

where the conditional density (on condition that the item did not fail in $[0, t)$ ) is

$$
\pi_{C}(z \mid t) \equiv \pi(z) \frac{\bar{F}_{C}(t, z)}{\int_{0}^{\infty} \bar{F}_{C}(t, z) \pi(z) d z} .
$$

As the failure rate under the transformed time scale is given by $\lambda_{C}(t, z)=c_{z}^{\prime}(t) \lambda\left(c_{z}(t), z\right)$, the result given in Proposition 1 still holds for the continuous mixing case. Thus, as in the discrete case, when the lifetime and efficiency are negatively associated, the efficiency based approach can change the shapes of the mixture failure rate completely. See also the next examples for more details.

Example 2. Suppose that $\lambda(t, z)=z \lambda, t \geq 0$, where $\lambda$ is a constant and $Z$ is exponentially distributed with parameter $\theta$. Then by direct integration in (3.2):

$$
\lambda_{m}(t)=\frac{\int_{0}^{\infty} z \lambda \exp \{-z \lambda t\} \theta \exp \{-\theta z\} d z}{\int_{0}^{\infty} \exp \{-z \lambda t\} \theta \exp \{-\theta z\} d z}=\frac{\lambda}{\theta+\lambda t},
$$

which is a decreasing function. Now, as $\lambda_{C}(t, z)=c_{z}^{\prime}(t) \lambda\left(c_{z}(t), z\right)=\left(z / e_{z}\right) \lambda, \lambda_{m C}(t)$ in (3.3) cannot be generally obtained in a closed form.

(Case I: Positive Association) Let $\lambda=1.0, \theta=0.5$, and $e_{z}=1 /(z+1), z \geq 0$. In this case, the graphs for $\lambda_{m}(t)$ and $\lambda_{m C}(t)$ are given in Figure 2 .

(Case II: Negative Association) Let $\lambda=1.0, \theta=0.5$, and $e_{z}=\exp \{z\}, z \geq 0$. In this case, the graphs for $\lambda_{m}(t)$ and $\lambda_{m C}(t)$ are given in Figure 3 .

In the following example, as in the discrete case, it can be seen that the consideration of the efficiency can change the shapes of the mixture failure rates dramatically.

Example 3. Consider the truncated extreme value distribution (Gompertz) defined in the following way:

$$
\begin{aligned}
\bar{F}(t, z) & =\exp \{-z k(\exp \{t\}-1)\}, \quad t \geq 0, \\
\lambda(t, z) & =z k \exp \{t\}, \quad z \geq 0, t \geq 0,
\end{aligned}
$$




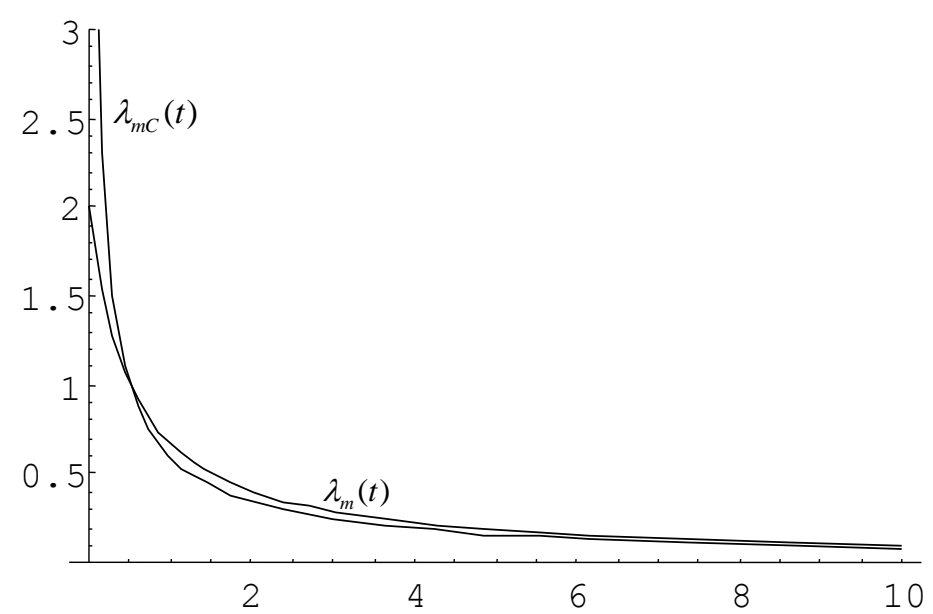

Figure 2: $\lambda_{m}(t)$ and $\lambda_{m C}(t)$

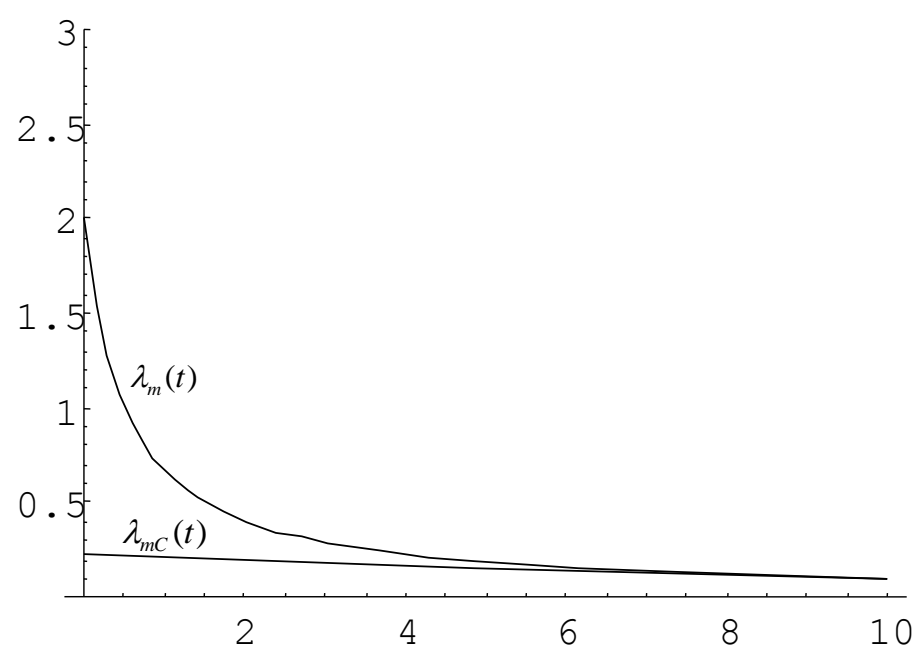

Figure 3: $\lambda_{m}(t)$ and $\lambda_{m C}(t)$

where $k>0$ is a constant. Let $Z$ be exponentially distributed with parameter $\theta$. Direct integration gives

$$
\begin{aligned}
\int_{0}^{\infty} f(t, z) \pi(z) d z & =\int_{0}^{\infty} z k \exp \{-z k(\exp \{t\}-1)\} \theta \exp \{-\theta z\} d z \\
& =\frac{\theta k \exp \{t\}}{\omega^{2}}
\end{aligned}
$$

where $\omega=k \exp \{t\}-k+\theta$ and

$$
\int_{0}^{\infty} \bar{F}(t, z) \pi(z) d z=\theta \int_{0}^{\infty} \exp \{-\omega z\} d z=\frac{\theta}{\omega} .
$$




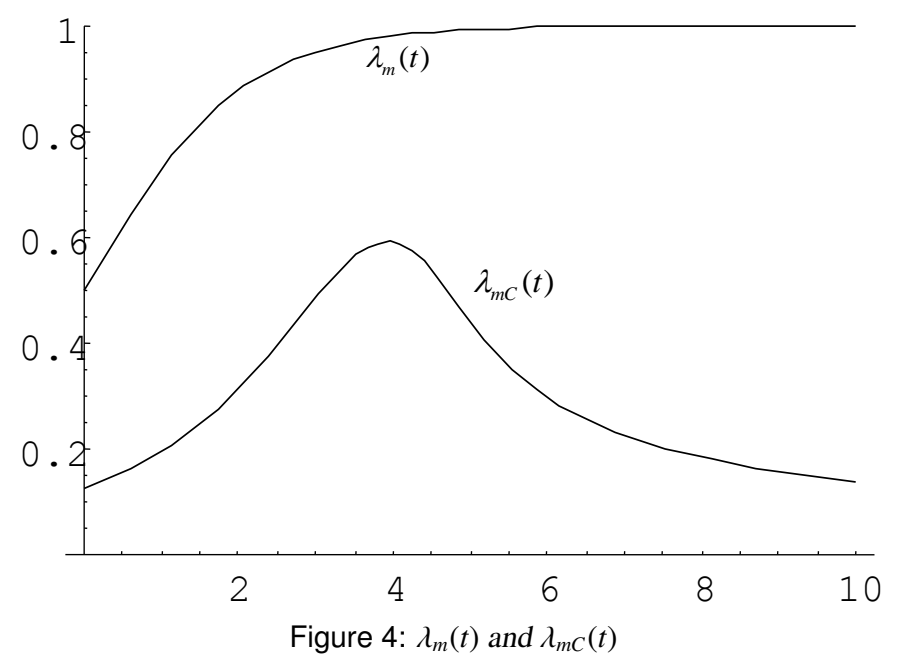

Eventually, using definition (3.2):

$$
\lambda_{m}(t)=\frac{k \exp \{t\}}{\omega}=1+\frac{k-\theta}{k \exp \{t\}-k+\theta} .
$$

Let $k<\theta$. Then $\lambda_{m}(t)$ is monotonically increasing asymptotically converging to 1 . Thus the baseline failure rate $k \exp \{t\}$ and the mixture failure rate $\lambda_{m}(t)$ are increasing. Let $k=0.5, \theta=1.0$ and $e_{z}=\exp \{z\}, z \geq 0$. In this case, the graphs for $\lambda_{m}(t)$ and $\lambda_{m C}(t)$ are given in Figure 4.

\section{Application to the Modeling of Environmental Effects}

In this section, we consider the application of the method of modeling efficiency based lifetime distributions studied in the previous section. In many instances, produced items are operated in different environmental stress levels. Thus, in this case, even though the baseline distribution is the same, their performances in field operation could be quite different and, accordingly, the environmental effect should be incorporated into the modeling of lifetime distribution. We discuss this topic in this section. For a proper formulation of the problem, we need to understand the modeling method commonly used in accelerated life tests(ALT).

Accelerated life tests are frequently used in practice to obtain timely information on the life distribution or performance over time of highly reliable products in an affordable amount of testing time. Test units are used more frequently than usual or are subjected to higher than usual levels of stress or stresses like temperature and voltage. Then the information obtained from the test performed in higher level of environment is used to predict actual product performance in usual level of environment. Nelson (1990) provides an extensive and comprehensive source for background material, practical methodology, basic theory, and examples for accelerated testing. Meeker and Escobar (1993) is also a good review paper of recent research and current issues in ALT. Denote random variable $X$ the lifetime of a component used in the usual level of environment and $F(t)$ the distribution function of $X$. We assume that $X$ is an absolutely continuous nonnegative random variable and thus the distribution has no probability mass at infinity. Let $f(t)$ be the probability density function of $X$. Its failure rate function $\lambda(t)$ is then given by $\lambda(t)=f(t) / \bar{F}(t)$, where $\bar{F}(t)$ is the survival function of $X$. Also denote 
random variable $X_{\mathcal{A}}$ the lifetime of a component operated in the accelerated level of environment and $F_{\mathcal{A}}(t), \lambda_{\mathcal{A}}(t)$ the corresponding distribution function and failure rate function, respectively. The 'Accelerated Failure Time(AFT)' regression model is the most widely used parametric failure time regression model in ALT. Under this model higher stress has the effect of shrinking time through a scale factor. This can be expressed as

$$
F_{\mathcal{A}}(t)=F(\rho \cdot t), \quad t \geq 0,
$$

where $\rho$ is a constant which depends on the accelerated stresses. As given in Section 3 of Meeker and Escobar (1993), a more general model can be expressed as

$$
F_{\mathcal{A}}(t)=F(\rho(t)), \quad t \geq 0,
$$

where $\rho(t)$ depends on the accelerated environment. Since the accelerated environment gives rise to higher stresses than the usual environment, reasonable assumptions are $\rho \geq 1$ for the model (4.1) and $\rho(t) \geq t$ for all $t \geq 0$ and $\rho(0)=0$ for the model (4.2). Furthermore we assume that $\rho(t)$ in the model (4.2) is strictly increasing, continuous and differentiable. Then, the models given in (4.1) and (4.2) imply that $X_{\mathcal{A}} \leq_{s t} X$. Here, the notation " $\leq_{s t}$ " denotes the usual stochastic order, that is, we say that $Z_{1}$ is said to be smaller than $Z_{2}$ in the usual stochastic order denoted as if $G_{2}(t) \leq G_{1}(t)$, for all $t \geq 0$, where $G_{1}(t)$ and $G_{2}(t)$ are the distribution functions of $Z_{1}$ and $Z_{2}$, respectively. From the model (4.2), the failure rate function in accelerated environment is given by

$$
\lambda_{\mathcal{A}}(t)=\frac{\rho^{\prime}(t) f(\rho(t))}{1-F(\rho(t))}=\rho^{\prime}(t) \lambda(\rho(t)) .
$$

Now we discuss our problem. Suppose that items produced constitute a homogeneous population. Suppose further that these items are operated in different stress levels of environment. Let the lifetime, distribution function, survival function, pdf and failure rate function of items operated in the "lowest stress level" of environment be denoted by $F(t), \bar{F}(t), f(t)$ and $\lambda(t)$ (baseline distribution). Let this stress level correspond to the frailty variable $Z=0$. In this case, the stress levels under which the items are operating can be regarded as continuous and these levels can be indicated using continuous variable $Z>0$. In this case, the larger value of $Z$ should correspond to the severer stress level of environment. Then applying the reasoning in ALT model, the distribution function which corresponds to the stress level with frailty $Z=z$ can be expressed as

$$
F(t, z)=F(\rho(t, z)), \quad z \geq 0, t \geq 0 .
$$

In model (4.3), $\rho(0, z)=0, \rho(t, 0)=t, \rho(t, z) \geq t$ for all $z \geq 0, t \geq 0$. Furthermore, $\rho(t, z)$ is strictly increasing in $z \geq 0$ and $t \geq 0$. Then, when the pdf of the frailty parameter $Z$ is given by $\pi(z)$, the lifetime distribution of items in field operation can be expressed using (3.1) and (3.2).

Example 4. Suppose that the baseline distribution is a Weibull distribution with $\lambda(t)=2 t$. Let $Z$ be exponentially distributed with parameter $\theta$ and $\rho(t, z)=\sqrt{z+1} \cdot t, z \geq 0, t \geq 0$. In this case, $F(t, z)=F(\rho(t, z))=1-\exp \left\{-(z+1) t^{2}\right\}$ and $\lambda(t, z)=2(z+1) t$. Then

$$
\pi(z \mid t) \equiv \pi(z) \frac{\bar{F}(t, z)}{\int_{0}^{\infty} \bar{F}(t, z) \pi(z) d z}=\left(\theta+t^{2}\right) \exp \left\{-\left(\theta+t^{2}\right) z\right\}, \quad z \geq 0 .
$$

Thus,

$$
\lambda_{m}(t)=\int_{0}^{\infty} \lambda(t, z) \pi(z \mid t) d z=2 t\left(\frac{1}{\theta+t^{2}}+1\right), \quad t \geq 0
$$




\section{Concluding Remarks}

In this paper, efficiency based reliability measures were defined when the performance of items in the mixture population has different levels. Discrete and continuous mixing models were considered. The concept of the association was defined and it was shown that the shape of the mixture failure rate changes dramatically when the negative association between the lifetime and the performance of items in the subpopulations exists. Useful applications to the cases with different stress levels of operating environment were also discussed.

In the reliability literature, the topics on burn-in procedure, warranty policy and maintenance policy have been discussed based on the ordinary reliability measures. However, as shown in this article, when the performance of items in subpopulations has different levels, the reliability measures should be defined in a different way and the obtained ageing property can be completely different. Therefore, in this case, the studies on burn-in procedure, warranty policy and maintenance policy should be done based on the reliability measures defined in this paper. These could be topics to be covered in future studies.

\section{Acknowledgement}

The author would like to thank the referees for helpful comments and suggestions, which have improved the presentation of this paper considerably.

\section{References}

Block, H. W., Savits, T. H. and Wondmagegnehu, E. T. (2003). Mixtures of distributions with increasing linear failure rates, Journal of Applied Probability, 40, 485-504.

Finkelstein, M. S. (2008). Failure Rate Modelling for Reliability and Risk, Springer, London.

Gupta, R. C. and Warren, R. (2001). Determination of change points of nonmonotonic failure rates, Communications in Statistics-Theory and Methods, 30, 1903-1920.

Jensen, F. and Petersen, N. E. (1982). Burn-in, John Wiley, New York.

Jiang, R. and Murthy, D. N. P. (1995). Modelling failure rate by mixture of two Weibull distributions. IEEE Transactions on Reliability, 44, 477-488.

Meeker, W. Q. and Escobar, L. A. (1993). A review of recent research and current issues of accelerated testing, International Statistical Review, 61, 147-168.

Navarro, J. and Hernandez, P. J. (2004). How to obtain bathtub-shaped failure rate models from normal mixtures, Probability in the Engineering and Informational Sciences, 18, 511-531.

Nelson, W. (1990). Accelerated Testing: Statistical Models, Test Plans, and Data Analysis, John Wiley \& Sons Inc, New York. 